\title{
Epigenetic modulation in the pathogenesis and treatment of inherited aortic aneurysm conditions
}

4 Authors: Benjamin E. Kang ${ }^{1}$, Rustam Bagirzadeh ${ }^{1}$, Djahida Bedja ${ }^{1}$, Jefferson J. Doyle ${ }^{1}$, Elena

5 G. MacFarlane ${ }^{1}$, Harry C. Dietz ${ }^{1,2 *}$

6 Affiliations:

$7{ }^{1}$ Department of Genetic Medicine, Johns Hopkins University School of Medicine, Baltimore, MD 821205 , USA.

$9 \quad{ }^{2}$ Howard Hughes Medical Institute, Chevy Chase, MD 20815, USA.

$11 *$ To whom correspondence should be addressed:

12 Hal C. Dietz

13 Professor, Institute of Genetic Medicine

14 Investigator, Howard Hughes Medical Institute

15 Johns Hopkins University School of Medicine

16 Miller Research Building Room 539

17733 N. Broadway

18 Baltimore, MD. 21205

19 hdietz@jhmi.edu

$20 \quad 410-614-0701$

22 Competing interests: B.E.K. and H.C.D are inventors on a patent application entitled "Targeted

23 Epigenetic Therapy For Inherited Aortic Aneurysm Condition,” (no. PCT/US2018/49217), jointly

24 filed on $31^{\text {st }}$ August 2018 by the Johns Hopkins University School of Medicine. All other authors

25 declare that they have no competing interests. 


\section{Abstract}

28 Shprintzen-Goldberg syndrome (SGS) is a rare systemic connective tissue disorder characterized

29 by craniofacial, skeletal, neurodevelopmental, cutaneous, and cardiovascular manifestations,

30 including aortic root aneurysm. It has significant phenotypic overlap with both Marfan syndrome

31 (MFS) and Loeys-Dietz syndrome (LDS). We previously reported that SGS is caused by

32 heterozygous mutations in the Sloan-Kettering Institute proto-oncogene (SKI), which encodes a

33 potent suppressor of transforming growth factor beta (TGF $\beta$ ) target gene expression. Herein, we

34 show that mouse lines harboring orthologous amino acid substitutions in Ski recapitulate multiple

35 human SGS phenotypic manifestations, including skin collagen deposition, skeletal kyphosis,

36 behavioral hypoactivity, and aortic root aneurysm. Furthermore, aortic root aneurysm in SGS mice

37 is associated with both increased acetylation of histone H3 at lysine-27 (H3K27) and TGF $\beta$ target

38 gene expression, all of which can be ameliorated by pharmacological CBP/P300 inhibition in vivo;

39 similar findings were seen in cultured dermal fibroblast from SGS patients. Aortic root growth is

40 also abrogated in a mouse model of MFS by selective CBP/P300 inhibition in association with

41 blunted expression of TGF $\beta$ target genes. These data document excessive H3K27 acetylation and

42 hence TGF $\beta$ target gene expression in the pathogenesis of inherited presentations of aortic root

43 aneurysm and the therapeutic potential of pharmacological epigenetic modulation. 
Introduction

Shprintzen-Goldberg syndrome (SGS) is a rare, autosomal dominant systemic connective

47 tissue disorder (CTD) characterized by craniosynostosis, severe skeletal deformities, aortic root

48 dilatation, minimal subcutaneous fat, intellectual disability, and neurodevelopmental anomalies (1,

49 2). It shows considerable phenotypic overlap in the craniofacial, skeletal, skin and cardiovascular

50 systems with both Marfan syndrome (MFS) and Loeys-Dietz syndrome (LDS), with the additional

51 findings of mental retardation and severe skeletal muscle hypotonia.

52 Mutations in the FBN1 gene encoding the extracellular matrix protein fibrillin-1 cause MFS,

53 while heterozygous mutations in the genes encoding TGF $\beta$ ligands (TGFB2 or TGFB3), receptor

54 subunits (TGFBR1 or TGFBR2) or intracellular signaling intermediates (SMAD2 or SMAD3) cause

$55 \operatorname{LDS}(3,4)$. This has led to the recognition that dysregulated TGF $\beta$ signaling plays a role in the

56 pathogenesis of both MFS and LDS $(4,5)$. In prior work, we have demonstrated increased

57 activation of both canonical (i.e. Smad2/3) and non-Smad (i.e. MAPK; Erk1/2) TGF $\beta$-dependent

58 signaling cascades in affected tissues of MFS and LDS mouse models (5-8). Furthermore, multiple

59 phenotypic manifestations in mice, including aortic root aneurysm, can be ameliorated by postnatal

60 administration of TGF $\beta$-neutralizing antibody, and/or the angiotensin II (Ang-II) type 1 (AT1)

61 receptor blocker (ARB) losartan, in association with blunted Smad2/3 and Erk1/2 activation (7, 9-

62 11). We also demonstrated that genetic ablation of Smad2 in cardiac neural crest-derived vascular

63 smooth muscle cells could abrogate aortic root aneurysm formation in mice with LDS caused by

64 a kinase domain mutation in $\operatorname{Tg} f b r l(12)$. This occurred in association with normalization of the

65 tissue signature for excessive TGF $\beta$ signaling in the aortic wall.

Despite these observations, there were a number of controversies that derived from this

67 initial work. First, certain features of SGS, including craniosynostosis, altered palatogenesis, and 
68 aortic aneurysm, have variably been associated with low TGF $\beta$ signaling states (13-15). This

69 raised the question as to whether low or high TGF $\beta$ signaling drives disease pathogenesis in SGS

70 and related CTDs. This has been further brought into question by the observation that LDS is

71 predominantly caused by heterozygous missense substitutions affecting the kinase domain of

72 either TGF $\beta R 1$ or TGF $\beta$ R2, that can cause a context-specific decline in TGF $\beta$ signal propagation

73 in cell culture systems $(4,16)$. Furthermore, LDS-like phenotypes are caused by heterozygous

74 loss-of-function mutations in $S M A D 3, T G F B 2$, or $T G F B 3$, all positive effectors of TGF $\beta$ signaling

$75(17,18)$. Once again, haploinsufficiency for these genes associated with a tissue signature for high

76 TGF $\beta$ signaling including excessive phosphorylation and nuclear translocation of Smad2/3 and

77 enhanced expression of typical TGF $\beta$ target genes including COL1A1, COL3A1, CTGF, MMP2

78 and $M M P 9(17,19,20)$. Taken together, these seemingly contradictory data have engendered

79 considerable controversy regarding the precise role of TGF $\beta$ signaling in the pathogenesis of

80 inherited forms of thoracic aortic aneurysm.

81 Given the extensive phenotypic overlap of SGS with both MFS and LDS, we hypothesized

82 that aberrant TGF $\beta$ activation likely underlies SGS and that identification of the genetic basis of

83 the syndrome would likely inform our understanding of both it and related CTDs. We and others

84 previously identified that de novo heterozygous mutations in the receptor-activated SMAD (R-

85 SMAD) binding domain of the Sloan-Kettering Institute proto-oncogene (SKI), a known repressor

86 of TGF $\beta$ signaling, cause SGS $(21,22)$. Furthermore, primary dermal fibroblasts from SGS

87 patients grown at steady-state showed a cell-autonomous increase in transcriptional output of many

88 TGF $\beta$-responsive genes. These data supported the conclusion that SGS pathogenesis appears to

89 be driven by high TGF $\beta$ signaling, presumably from loss of suppression by mutant SKI protein. It 
90 remains unclear, however, whether these mutations in SKI drive SGS pathogenesis via a loss-of-

91 function, dominant-negative, or gain-of-function mechanism.

Because of the plethora of SKI binding partners, the downstream consequences of $S K I$

93 mutations remain uncertain. SKI appears to bind to an array of partners, including R-SMADs

94 (SMAD2 and SMAD3), SMAD4, SKI itself (during dimerization), as well as SKI-like peptide

95 (SKIL), and transcription factors such as CBP/P300, mSin3A, SNW1, N-CoR and HDAC1 (23-

96 30). SGS-causing mutations have to date clustered in the R-SMAD binding domain of SKI towards

97 the N-terminal end of the protein, including substitution of an amino acid that has previously been

98 shown to be essential for SKI-SMAD3 interactions (e.g. p.Leu21Arg) (30). Interestingly, a version

99 of SKI lacking this R-SMAD binding domain retained its ability to regulate SMAD-mediated transcriptional activation in a transient transfection-based reporter system, but failed to dissociate

$101 \mathrm{CBP} / \mathrm{P} 300$ from the SMAD complex $(28,30)$, suggesting that CBP/P300 could play a role in SGS.

102 More recently, it was shown that regulated degradation of SKI requires interaction with SMAD2

103 or SMAD3 and SMAD4; SGS mutations that prevent SMAD2/3 binding resulted in increased

104 stability and hence abundance of mutant SKI, which retained the ability for transcriptional

105 repression of some TGF $\beta$ target genes, as evidenced by reduced induction of these transcripts in

106 cultured cells expressing mutant SKI to acute stimulation with exogenous TGF $\beta$ (31). Many

107 questions remain regarding the potentially opposing influences of different aspects of altered SKI

108 homeostasis in SGS including increased stability and abundance that is perhaps offset by altered

109 efficiency for recruitment to regulatory elements of target genes. Given that SGS-causing

110 mutations in SKI protein interfere with binding to pSMAD2 and pSMAD3, altered transcriptional

111 regulation is presumably mediated through interaction with SMAD4 only and by aberrant

112 regulation of epigenetic modulators such as CBP/P300. The net effect of heterozygous SGS 
113 mutations on TGF $\beta$ signaling may vary based on cell type, baseline signaling status, redundancy

114 of autoregulatory factors, and the potential for chronic compensatory events.

115 In an attempt to inform these questions, we generated and characterized a knock-in mouse

116 model of SGS and explored the molecular mechanisms driving disease pathogenesis in these

117 animals. We used this information to develop a novel therapeutic strategy for the disorder, which

118 also shows efficacy in a well-characterized mouse model of MFS. Finally, we show concordant

119 molecular events and therapeutic potential in cultured dermal fibroblasts from patients with SGS. 


\section{Results}

122

123

124

125

126

127

128

129

130

131

132

133

134

135

136

137

138

139

140

141

142

143

SGS mouse models recapitulate the phenotype of patients with SGS

To determine whether mutations in SKI are sufficient to recapitulate the SGS phenotype in mice, several targeted mouse lines were developed (Fig. 1A). $S k i^{+/-}$mice are heterozygous for a deletion of exons 2 and 3 of Ski, which leads to functional haploinsufficiency due to nonsensemediated mRNA decay. $S k i^{\mathrm{G} 34 \mathrm{D} /+: \mathrm{Neo}}$ mice are heterozygous for a missense mutation (p.Gly34Asp) previously observed in a patient with severe SGS (21); this allele retains the neomycin resistance cassette, which causes transcriptional interference and also leads to functional haploinsufficiency. $S k i^{\mathrm{G} 34 \mathrm{D} /+}$ mice are generated by breeding of $S k i^{\mathrm{G} 34 \mathrm{D} /+: \text { Neo }}$ mice to transgenic mice expressing an ubiquitous Cre recombinase (CMV-Cre), which eliminates the Neo cassette, thus allowing transcription of the mutant allele and expression of mutant SKI. As expected, both haploinsufficient lines expressed half the normal complement of Ski mRNA, while the heterozygous $S k i^{\mathrm{G} 34 \mathrm{D} /+}$ line expressed significantly higher levels of $S k i$ mRNA, to levels indistinguishable from control mice (Fig. 1B). The $S k i^{\mathrm{G} 34 \mathrm{D} /+}$ mouse line uniquely expressed the mutant allele, as shown by a diagnostic BamH1 restriction fragment of amplified cDNA that was present only in this line (Fig. 1C).

Mice heterozygous for the mutant allele $\left(S k i^{\mathrm{G} 34 \mathrm{D} /+}\right)$ recapitulated multiple phenotypic characteristics of patients with SGS. In vivo echocardiography of these mice showed evidence of increased aortic root size at 6 months of age and enhanced post-natal aortic root growth from 2 to 6 months of age, compared to wild-type (WT) littermates (Fig. 1D, S1A). They also showed evidence of skeletal deformity in the form of spine kyphosis (Fig. 1F), as well as reduced subcutaneous fat and increased collagen deposition in the skin (Fig. S1B, C), at 6 months of age, in comparison to WT littermates. Finally, they displayed abnormal behavior including hypoactivity 
144 and impaired motor performance at 10 weeks of age, when compared to WT littermates (Fig. S1D,

145 E). In contrast, mice haploinsufficient for $S k i\left(S k i^{+/-}\right.$or $\left.S k i^{\mathrm{G} 34 \mathrm{D} /+: \mathrm{Neo}}\right)$ showed no evidence of these

146 phenotypic defects when compared to WT littermates.

147 We bred our conditional G34D allele $\left(S k^{\mathrm{G} 34 \mathrm{D} /+ \text { :Neo }}\right)$ mouse line to transgenic mice carrying

148 the vascular smooth muscle cell (VSMC) specific Sm22 $\alpha$-Cre driver (32), to generate mice that

149 selectively expressed mutant SKI only in VSMC populations. These mice $\left(S k i^{\mathrm{VSMC}: G 34 \mathrm{D} /+}\right)$ also

150 showed evidence of aortic root aneurysm at 6 months of age compared to WT littermates, and

151 increased post-natal aortic root growth between 2 and 6 months of age, that was indistinguishable

152 from the rate of growth seen in $\mathrm{Ski}^{\mathrm{G} 34 \mathrm{D} /+}$ mice, which ubiquitously express mutant Ski (Fig. 1D, 153 S1A).

As previously observed in dermal fibroblasts from patients with SGS (21), qPCR of aortic

155 tissue taken from $S k i^{\mathrm{G} 34 \mathrm{D} /+}$ and $S k i^{\mathrm{VSMC}: \mathrm{G} 34 \mathrm{D} /+}$ mice showed increased expression of all assayed 156 TGF $\beta$ target genes, including Col3A1, Fn1, Smad7, Mmp2, Col1A1, Cdkn1a, Ctgf, Serpine1, Skil,

157 and Mmp9 (encoding type 3 collagen, fibronectin, SMAD family member 7, matrix 158 metallopeptidase 2, type 1 collagen, p21, connective tissue growth factor, PAI-1, SKI-like protein, 159 and matrix metallopeptidase 9, respectively; Fig. 1E, S2).

160 These data confirm that this knock-in mouse model of SGS recapitulates multiple 161 phenotypic manifestations of the disorder seen in humans, and that the aortic root aneurysm seen

162 in these mice associates with increased TGF $\beta$-dependent target gene expression in the aortic wall.

163 The absence of a phenotype in $S k i^{\mathrm{G} 34 \mathrm{D} /+: \mathrm{Neo}}$ mice supports the conclusion that the SGS phenotype

164 does not manifest as a result of Ski haploinsufficiency, leaving open the possibility of either a 165 dominant negative mechanism of action or a novel gain of function. 

mice

The ARB losartan has previously been shown to ameliorate aortic root aneurysm progression in mouse models of both MFS and LDS, in association with blunted Smad2/3 and

171 Erk1/2 activation (8, 9). To investigate the potential therapeutic effect of losartan in SGS, 2-month

172 old $S k i^{\mathrm{G} 34 \mathrm{D} /+}$ and $S k i^{\mathrm{VSMC}: G 34 \mathrm{D} /+}$ mice were treated for 4 months with a dose of losartan previously

173 shown to be efficacious in MFS and LDS mice. Aortic root size was measured at 2 months of age

174 (pre-treatment baseline) and every month thereafter until 6 months of age. Aortic root growth was 175 significantly greater in placebo-treated $S k i^{\mathrm{G} 34 \mathrm{D} /+}$ and $S k i^{\mathrm{VSMC}: \mathrm{G} 34 \mathrm{D} /+}$ mice compared with WT 176 littermates (Fig. 2A, S3A).

178 significant reduced in losartan-treated $S k i^{\mathrm{G} 34 \mathrm{D} /+}$ and $S k i^{\mathrm{VSMC}: \mathrm{G} 34 \mathrm{D} /+}$ mice to ate indistinguishable

179 from that observed in control mice. No significant change in total body weight was observed with losartan treatment (Fig. S3B). ARBs such as losartan lower blood pressure, which is known to be

181 beneficial in slowing aortic aneurysm growth. To investigate whether losartan was achieving its 182 protective effect solely through blood pressure reduction, we performed a head-to-head trial with 183 another antihypertensive agent, the beta-blocker atenolol. Ski ${ }^{\mathrm{G} 34 \mathrm{D} /+}$ and $S k i^{\mathrm{VSMC}: \mathrm{G} 34 \mathrm{D} /+}$ mice and 184 control littermates were treated with hemodynamically-equivalent doses of either atenolol $185(60 \mathrm{mg} / \mathrm{kg} /$ day $)$ or losartan $(50 \mathrm{mg} / \mathrm{kg} /$ day $)$, from 2 to 6 months of age. Aortic root growth during 186 this 4 month period in atenolol-treated $S k i^{\mathrm{VSMC}: \mathrm{G} 34 \mathrm{D} /+}$ mice was significantly less than that of 187 placebo-treated $S k i^{\mathrm{VSMC}^{\mathrm{G} 34 \mathrm{D} /+}}$ mice (Fig. S3C), as expected following blood pressure reduction. 188 By contrast, losartan-treated $S k i^{\mathrm{VSMC}: \mathrm{G} 34 \mathrm{D} /+}$ mice showed a significantly greater reduction in aortic 
190 reduction in blood pressure and no significant change in total body weight with the 2 drugs (Fig. 191 S3C, D, E) Histological and morphometric analyses of aortic wall cross sections were performed

193 following death or sacrifice of the mice at 6 months of age. Verhoeff-Van Gieson (VVG) and 194 trichrome staining displayed evidence of aortic wall thickening due to massive accumulation of 195 collagen in the aortic media, reduced elastin content, and increased elastic fiber fragmentation in 196 placebo-treated $S k i^{\mathrm{G} 34 \mathrm{D} /+}$ and $S k i^{\mathrm{VSMC}: \mathrm{G} 34 \mathrm{D} /+}$ mice, compared with WT littermates, all of which were 197 normalized by losartan treatment (Fig. 2B, C, D).

To further confirm that losartan was achieving its effect through a mechanism other than 199 simple blood pressure reduction, we assessed TGF $\beta$-dependent target gene expression in the aortas 200 of 6-month old mice. Placebo-treated $S k i^{\mathrm{G} 34 \mathrm{D} /+}$ mice and $S k i^{\mathrm{VSMC}: \mathrm{G} 34 \mathrm{D} /+}$ mice showed a significantly 201 greater expression of TGF $\beta$-dependent target genes, compared with WT littermates (Fig. 2E, S2).

202 By contrast, losartan treatment led to a significant reduction in the expression of these genes in $203 S k i^{\mathrm{G} 34 \mathrm{D} /+}$ mice and $S k i^{\mathrm{VSMC}: \mathrm{G} 34 \mathrm{D} /+}$ mice, to levels indistinguishable from those seen in WT 204 littermates in most instances. These data support the conclusion that ARB blockade with the use 205 of losartan appears highly efficacious in treating aortic aneurysm growth in knock-in mouse 206 models of SGS, in association with reduced TGF $\beta$-dependent gene expression, analogous to what 207 has been observed in mouse models of both MFS and LDS (8, 9). It may hence represent a novel 208 therapeutic strategy for the treatment of patients with SGS.

The G34D mutation is located in the R-SMAD binding domain of SKI. Interestingly, a 212 mutated form of SKI lacking this R-SMAD binding domain was found to retain its ability to 
213 regulate SMAD-mediated transcriptional activation in a transient transfection reporter assay, but

214 failed to dissociate CBP/P300 from the SMAD complex $(28,30)$. Indeed, SKI and CBP/P300 are

215 known to compete for binding to R-SMADs, and binding of SKI to R-SMADs is sufficient to

216 displace CBP/P300, an effect that is not mimicked by the interaction of SKI with SMAD4.

217 Maintenance of CBP/P300 within the complex promotes gene expression via increased H3K27

218 acetylation and hence preservation of an open chromatin state (33-39). An increase is resident

$219 \mathrm{CBP} / \mathrm{P} 300$ can also positively regulate transactivation activity through acetylation of the MH2

220 domain of SMAD3 at lysine 378 (40). Our prior phenotyping data confirmed that

221 haploinsufficiency for SKI does not appear to be the mechanism of action in SGS. We therefore

222 hypothesized that SGS-causing missense mutations in the R-SMAD binding domain of SKI might

223 allow maintenance of $\mathrm{CBP} / \mathrm{P} 300$ binding despite residual recruitment of SKI to regulatory

224 elements in target genes via interaction with SMAD4. The resulting increase in H3K27 acetylation

225 and heightened and/or prolonged TGF $\beta$-dependent gene transcription would not manifest in

226 experimental systems that are not susceptible to this type of epigenetic regulation.

227

To investigate this, we first performed immunofluorescence staining to look for evidence of

228 enhanced $\mathrm{H} 3 \mathrm{~K} 27$ acetylation in the aortic root of $S k i^{\mathrm{VSMC}: \mathrm{G} 34 \mathrm{D} /+}$ mice at 6 months of age. Compared

229 with control littermates, Ski $i^{\mathrm{VSMC}: \mathrm{G} 34 \mathrm{D} /+}$ mice did indeed show much greater H3K27 acetylation in

230 the medial layer of the aortic root (Fig. 3A). To confirm that increased H3K27 acetylation is a

231 driver of aortic aneurysm progression in SGS, rather than simply a marker of it, we treated

$232 S k i^{\mathrm{VSMC}: G 34 \mathrm{D} /+}$ mice with the selective CBP/P300 inhibitor C646 (41). Treatment with C646 (1

$233 \mathrm{mg} / \mathrm{kg} /$ day) was started at 2 months of age and continued for 3 months until sacrifice of the mice

234 at 5 months of age. Control mice were treated with the vehicle dimethyl sulfoxide (DMSO) alone.

235 Aortic root size was measured at 2 months of age (pre-treatment baseline) and every month 
236 thereafter. Aortic root growth during the treatment period was significantly greater in vehicle-

237 treated $S k i^{\mathrm{VSMC}: \mathrm{G} 34 \mathrm{D} /+}$ mice, compared with control littermates (Fig. 4A, S4A), and was comparable

238 to that previously seen in placebo-treated $S k i^{\mathrm{VSMC}: \mathrm{G} 34 \mathrm{D} /{ }^{+}}$mice. By contrast, C646 treatment led to

239 a significant reduction in aortic root growth in $S k i^{\mathrm{VSMC}: \mathrm{G} 34 \mathrm{D} /{ }^{+}}$mice, to a rate indistinguishable from

240 that observed in control mice, without a change in total body weight (Fig. S4B). Importantly, C646

241 therapy had no effect in control mice, showing that inhibition of H3K27 acetylation was selectively

242 targeting Ski mutation-associated pathological aortic root growth rather than physiological aortic

243 growth.

244 The specificity of C646 was confirmed by immunofluorescence staining for H3K27

245 acetylation. Compared with DMSO-treated Ski $i^{\mathrm{VSMC}: G 34 \mathrm{D} /+}$ littermates, C646-treated SkiVSMC:G34D/+

246 mice showed a clear reduction in H3K27 acetylation in the medial layer of the aortic root (Fig.

247 3A). Histological and morphometric analyses of aortic wall cross sections were performed

248 following death or sacrifice of the mice at 5 months of age. VVG and trichrome staining

249 reconfirmed prominent aortic wall thickening, reduced elastin content, and increased elastic fiber

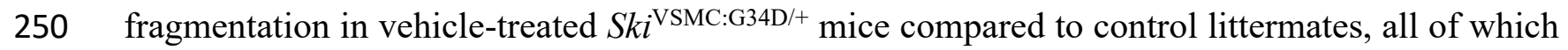

251 were significantly improved in C646-treated $S k i^{\mathrm{VSMC}: G 34 \mathrm{D} /+}$ mice (Fig. 3B, C, D).

252 To determine whether this reduction in H3K27 acetylation by C646 directly impacted

253 TGF $\beta$-dependent gene expression in the aortas of these mice, we performed qPCR on the aortas

254 of 5-month old mice. The increased expression of TGF $\beta$ target genes seen in vehicle-treated

$255 S k i^{\mathrm{VSMC}: \mathrm{G} 34 \mathrm{D} /+}$ mice was indeed significantly reduced in C646-treated $S k i^{\mathrm{VSMC}: \mathrm{G} 34 \mathrm{D} /+}$ mice, to

256 levels close to or indistinguishable from those seen in control mice (Fig. 4B, S5). To confirm that

257 C646 does not have any antihypertensive effect, we measured blood pressure in these animals and 
258 found no significant difference between vehicle-treated $S k i^{\mathrm{VSMC}: G 34 \mathrm{D} /+}$ mice and C646-treated $259 S k i^{\mathrm{VSMC}: \mathrm{G} 34 \mathrm{D} /+}$ littermates (Fig. S4C).

261 CBP/P300 activity, increased H3K27 acetylation, and ultimately prolonged TGF $\beta$-dependent gene

262 expression, all of which can be ameliorated in a mouse model of SGS by selective CBP/P300

263 inhibition. Since animal models of MFS, in particular the $F b n 1^{\mathrm{C} 1039 \mathrm{G} /+}$ mouse model, also show

264 increased TGF $\beta$-dependent gene expression in their aortas, we hypothesized that selective

$265 \mathrm{CBP} / \mathrm{P} 300$ inhibition could represent a novel therapeutic strategy for other forms of inherited aortic

266 aneurysm. To assess this, we treated $\mathrm{Fbnl}^{\mathrm{C} 1039 \mathrm{G} /+}$ mice with C646 from 2 months of age for 2

267 months. Aortic root growth over the treatment period was significantly greater in vehicle-treated

$268 \mathrm{Fbnl}^{\mathrm{C1039 \textrm {G } / +}}$ mice than WT littermates (Fig. 4C). By contrast, C646 treatment led to a significant

269 reduction in aortic root growth in $\mathrm{Fbnl}^{\mathrm{C} 1039 \mathrm{G} /{ }^{+}}$mice, to a rate comparable to that observed in WT

270 controls. This reduction in aortic root growth was associated with a significant reduction in TGF $\beta-$

271 dependent gene expression in C646-treated $\mathrm{Fbnl}^{\mathrm{C} 1039 \mathrm{G} /{ }^{+}}$mice, compared to their vehicle-treated

272 littermates (Fig. 4D).

$274 C B P / P 300$ inhibition abrogates TGF $\beta$-dependent gene expression in SGS patient cells

275 We tested the effect of C646 on primary dermal fibroblasts derived from 2 SGS patients and

2762 healthy controls. Vehicle-treated fibroblasts from SGS patients showed significantly greater

277 mRNA expression of TGF $\beta$-dependent genes, including COL3A1, FN1, SKI, and SMAD7,

278 compared with control fibroblasts, both at baseline and after stimulation with exogenous TGF $\beta 1$

$279(10 \mathrm{ng} / \mathrm{ml})$ for 6 hours (Fig. S6). By contrast, C646 treatment of SGS patient fibroblasts led to a

280 significant reduction in expression of these genes, to levels similar to those seen in control 
281 fibroblasts, or SGS patient fibroblasts treated with the TGF $\beta$ type I receptor (Alk5) kinase inhibitor

282 SD208. Selective CBP/P300 inhibition thus appears to suppress TGF $\beta$-dependent gene expression

283 in both mouse models of SGS and cells derived from patients with SGS. 


\section{Discussion}

Shprintzen-Goldberg syndrome (SGS) in a rare systemic connective tissue disorder caused

287 by heterozygous mutations in the $S K I$ gene, and shows significant phenotypic overlap with both

288 MFS and LDS. Despite the initial discovery of a causal gene, a number of questions remained unanswered about the disorder, including the effect of SGS-causing mutations on TGF $\beta$ signaling

290 in vivo, the exact role of TGF $\beta$ signaling in the disease, and which SKI binding partners and

291 downstream signaling sequelae contribute to pathogenesis.

292

The body of evidence implicating dysregulation of TGF $\beta$ signaling in vascular connective

293 tissue disorders is extensive and compelling. Virtually every study of these conditions has provided

294 evidence for high TGF $\beta$ signaling in the aortic wall of mouse models or people with MFS or LDS,

295 including enhanced activation of signaling intermediates (i.e. phosphorylation and/or nuclear

296 translocation of SMAD2/3) and high output of TGF $\beta$ target genes in relevant tissues. Yet

297 ambiguity is demonstrable and controversy substantial. Evidence suggested that fibrillin-1, the

298 deficient gene product in Marfan syndrome, could positively regulate TGF $\beta$ signaling by

299 concentrating cytokine at sites of intended function, but negatively regulate signaling by

300 sequestering the TGF $\beta$ latent complex from activators (5, 42-44). All forms of LDS are caused

301 by heterozygous loss-of-function mutations in genes encoding positive effectors of TGF $\beta$

302 signaling $(4,17,45-47)$. Therapeutic trials were equally ambiguous. While the consistent

303 therapeutic benefit of ARBs in mouse models of MFS or LDS strictly correlated with attenuation

304 of the tissue signature for high TGF $\beta$ signaling in the aortic wall, administration of TGF $\beta$

305 neutralizing antibodies accentuated disease in the perinatal period in MFS mice, while affording

306 significant protection later in postnatal life, with heightened efficacy when used in combination

307 with ARBs (9). The fact SGS includes essentially every systemic manifestation of MFS (except 
lens dislocation) and LDS seemed particularly relevant given the prominent role of SKI in the negative regulation of the TGF $\beta$ transcriptional response $(1,2,21,48)$. The observations that SGS mutations clustered in the R-SMAD binding domain of SKI, and that SGS patient fibroblasts showed high expression of TGF $\beta$-responsive genes known to contribute to aneurysm progression suggested that increased TGF $\beta$-dependent events may cause the multisystem manifestations of SGS. This might also inform the mechanism for similar manifestations in MFS and LDS. enhanced stability of SKI, and that this was associated with decreased expression of selected TGF $\beta$ target genes in either cells transfected with mutant SKI or in SGS fibroblasts (31). Notably, this

317 study only assessed the acute phase-response to administered TGF $\beta$ ligand (1 and 8 hours after delivery), and focused on genes that are predominantly expressed in neurons or polarized epithelial

319 cells, but not the aortic wall or dermal fibroblasts. Prior work had demonstrated that loss of binding of SKI to R-SMADs, as imposed by SGS mutations, did not abrogate the regulation of the TGF $\beta$ transcriptional response in reporter allele assays, but rather impaired the ability of SKI to displace

$322 \mathrm{CBP} / \mathrm{P} 300$ at critical regulatory elements, potentially altering the efficiency of chronic SKI-

323 mediated termination of a signal initiated by $\operatorname{TGF} \beta(28,30)$. This effect would be best interrogated

324 upon chronic exposure to TGF $\beta$, with potentially unique insights afforded by the study of affected 325 tissues in vivo. Moreover, the impact of an SGS mutation could vary based upon cell type, with 326 particular relevance for the expression of redundant negative regulators (e.g. SKIL or SMAD7) or 327 factors involved in chronic compensation. Importantly, our prior studies in dermal fibroblasts 328 derived from SGS patients assessed TGF $\beta$ target gene expression at steady-state, with no overlap 329 between the repertoire of genes previously assessed and those specifically examined by Hill and 330 colleagues $(21,31)$. We now unequivocally show that constitutive or VSMC-specific expression 
331 of a heterozygous SGS-associated SKI mutation leads to a substantial and sustained increase in

332 H3K27 acetylation in vivo, as predicted by increased CBP/P300 occupancy, in association with

333 high expression of TGF $\beta$ target genes relevant to aortic disease and reliably assayed in dermal

334 fibroblasts. These findings correlated with the excessive accumulation of fibrillar collagens in the

335 vessel wall and skin of SGS mouse models, as predicted by amplification of the TGF $\beta$

336 transcriptional response, with notable downregulation appearing coincident with therapeutic

337 interventions that achieved attenuation of aneurysm progression.

These data are consistent with prior work showing that CBP/P300-mediated H3K27

339 acetylation is enriched in the promoter regions of TGF $\beta$ target genes (49). Furthermore,

$340 \mathrm{CBP} / \mathrm{P} 300-$ mediated histone acetylation at the PAI-1 and p21 promoters can enhance TGF $\beta 1$ -

341 induced expression of these genes in cell culture systems (50). By contrast, CBP/P300 inhibition

342 using C646 has been shown to significantly reduce H3K27 acetylation (51), and also abrogate

343 expression of a number of TGF $\beta$ target genes, including Cdkn la, Mmp, Serpinel and Ctgf (52-

344 55). Prior work has shown that C646 can have a substantial effect on behavioral characteristics in

345 rodents such fear memory $(56,57)$, and on cardiac fibrosis and hypertrophy in Sirt3 deficient mice

346 (58). Although we have shown efficacy of C646 against aortic aneurysm progression in SGS and

347 MFS mice, its therapeutic potential for other systemic manifestations of these vascular connective

348 tissue disorders remains untested.

ARBs such as losartan have previously been shown to attenuate TGF $\beta$ signaling through

350 downregulation of TGF $\beta$ ligands, receptors and activators such as PAI-1 $(9,11)$. The overt

351 protection from aneurysm progression seen in mouse models of MFS and LDS associates with a

352 reduction in TGF $\beta$ signaling in the aortic root media, as evidenced by normalization of SMAD2/3

353 phosphorylation and the expression of TGF $\beta$ target genes in the aortic wall, prominently including 
354 fibrillar collagens, MMPs 2 and 9 and CTGF $(7,8,11)$. A role for losartan in epigenetic modulation of the TGF $\beta$ transcriptional response is less clear. Work by Reddy et al. has shown

356 that losartan can ameliorate diabetic nephropathy in mice through a reduction in H3K9/14

357 acetylation at the promoters of pathogenic genes (59). Furthermore, losartan was found to

358 attenuate proteinuric kidney disease in mice via inhibition of DNA methylation at the nephrin

359 promoter (60). The relative importance of each of these potential mechanisms of action for ARBs

360 in SGS remains to be determined. Our current hypothesis is that ARBs predominantly suppress

361 early events in TGF $\beta$ signaling, precluding abnormal maintenance and/or amplification of the

362 transcriptional response in the context of a SKI functional deficiency.

Given the known phenotypic overlap, and the newly-confirmed biochemical similarity,

364 between SGS and both MFS and LDS, a logical extension of this work was to elucidate whether

365 epigenetic changes and their pharmacological manipulation may hold relevance to other CTDs.

366 Indeed, treatment with C646 did lead to a significant reduction in aortic root growth in MFS mice,

367 in association with reduced TGF $\beta$ target gene expression in the aortic wall. This suggests that a

368 reduction in $\mathrm{H} 3 \mathrm{~K} 27$ acetylation can achieve a therapeutic effect, likely by facilitating a more

369 closed chromatin state that impairs TGF $\beta$ target gene transcription, despite the presence of

370 increased upstream TGF $\beta$ signaling. The findings of this study suggest that epigenetic modulation

371 holds potential for the treatment in diverse presentations of syndromic forms of aortic aneurysm.

372 These data add to the extensive and compelling in vivo evidence for enhanced TGF $\beta$

373 signaling in the pathogenesis of vascular connective tissue disorders including MFS, LDS and SGS,

374 with particular emphasis on the vascular pathology. In comparison, there is no documented

375 example of decreased TGF $\beta$ signaling in tissues derived from people or mouse models of these

376 conditions. Yet, we view the evidence for primary functional impairment of the TGF $\beta$ signaling 
377 response by underlying mutations to be equally compelling, including the evidence by Hill and

378 colleagues that SGS mutations can stabilize SKI and associate with relative impairment of

379 transcriptional responses in selected culture systems (31). The challenge - and, we would argue,

380 the opportunity - lies in embracing all inconvenient truths to arrive at reconciling and testable

381 models that have the true potential to inform disease pathogenesis and therapeutic opportunities.

382 In the case of LDS, we have shown that heterozygous loss-of-function mutations in positive

383 effectors of TGF $\beta$ signaling have a disproportionate and at times even unique negative impact on

384 responses in specific cell types (12). This can lead to paracrine effects (e.g enhanced TGF $\beta$ ligand

385 production) that drive excessive signaling in neighboring cell types that are less vulnerable to the

386 consequences of underlying mutations (12). Given the potency and redundancy of mechanisms for

387 autoregulation of TGF $\beta$ signaling, it seems possible that the apparent low signaling - high

388 signaling paradox in the TGF $\beta$ vasculopathies is actually a requirement for the initiation and

389 maintenance of a high TGF $\beta$ signaling state. There is ample precedent for this in the TGF $\beta$ cancer

390 paradox, where TGF $\beta$ serves both as a tumor suppressor and a positive regulator of tumor

391 progression. We anticipate that consideration and integration of this physiologic complexity will

392 be required to achieve consensus - and truth - in our field. 
Methods

Mouse lines

All mice were cared for under strict compliance with the Animal Care and Use Committee

397 of the Johns Hopkins University School of Medicine. Ski G34D/+ mice were generated by

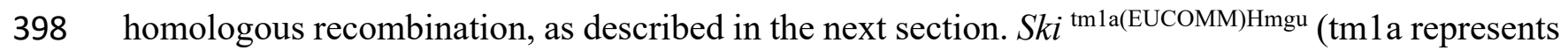

399 targeted mutation 1a, and hmgu represents Helmholtz Zentrum Muenchen GmbH) embryonic stem

400 cells were obtained from the European Conditional Mouse Mutagenesis Program and injected into

401 the cavity of day 3.5 blastocysts from C57BL/6J mice at the Johns Hopkins University School of

402 Medicine transgenic core. Male chimeras were mated with C57BL/6J WT female mice to establish

403 germline transmission. The LacZ-Neo cassette was removed by crossing with a FlpO transgenic

404 strain (B6.Cg-Tg(Pgk1-flpo)10Sykr/J, \#011065) purchased from the Jackson Laboratory and to

405 generate $S k i^{+/-}$mice, exon 2 and 3 of $S k i$ gene flanking by loxP sequences were removed by

406 crossing with a transgenic Cre strain (B6.C-Tg(CMV-cre)1Cgn/J, \#006054) purchased from the

407 Jackson Laboratory, followed by mating to the C57BL/6J strain at least 5 generations. Sm $22 \alpha-\mathrm{Cre}$

408 mice (B6.Cg-Tg(Tagln-cre)1Her/J, \#017491) were purchased from the Jackson Laboratory,

409 followed by mating to the $\mathrm{C} 57 \mathrm{BL} / 6 \mathrm{~J}$ strain for at least 5 generations. To minimize potentially

410 confounding background effects, all comparisons between genotypes and between treatment arms

411 within a genotype were made between gender-matched littermates.

Mice were checked daily for evidence of premature lethality. At the end of a drug trial, all

413 mice were euthanized through inhalational halothane (Sigma) or anesthetized with isofluorane.

414 Following sacrifice, mice underwent immediate laparotomy, descending abdominal aortic

415 transection, and phosphate-buffered saline (PBS; $\mathrm{pH}$ 7.4) was infused throughout the vascular tree 416 via the left ventricle. For the aorta frozen section, additional 4\% paraformaldehyde in PBS was 
417 infused again for fixation tissue. Sacrificed mice used for 3 harvest methods, latex infusion for

418 histological analysis, freezing heart and aorta embedded in optimal cutting temperature compound

419 (O.C.T compound) for immunofluorescent staining, and in-situ hybridization and snap-frozen

420 aorta in liquid nitrogen.

421 For quantitative RT-PCR, the aortic root and ascending aorta (above the aortic root to the

422 origin of right brachiocephalic trunk) of mice were harvested separately, snap-frozen in liquid

423 nitrogen, and stored at $-80^{\circ} \mathrm{C}$ until processed. For RNA extraction, aortas were homogenized in

424 TRizol (ThermoFisher) by FastPrep-24 (MP Biomedicals, LLC), per the manufacturer's

425 instructions. After homogenization, RNA was extracted using an RNeasy mini kit (QIAGEN), per

426 the manufacturer's instructions. The RNA samples were then stored once more at $-80^{\circ} \mathrm{C}$ until

427 quantitative RT-PCR was performed.

428 For the frozen aorta sections, aorta with heart was harvested and fixed in fresh $4 \%$ 429 paraformaldehyde in $\mathrm{PBS}$ at $4^{\circ} \mathrm{C}$ overnight, and then placed in cold $30 \%$ sucrose in PBS solution 430 and incubated at $4^{\circ} \mathrm{C}$ overnight again. Tissue was then embedded in Tissue-Tek O.C.T compound 431 and snap-frozen in liquid nitrogen, and stored at $-80^{\circ} \mathrm{C}$ until processed.

433 were analyzed for aortic histology had latex infused into the left ventricle through the descending 434 abdominal aorta. Mice were then fixed for 24 hours in 10\% neutral-buffered formalin, before being 435 stored in 70\% ethanol until the histological analysis was performed.

437 Generation of Ski ${ }^{G 34 D /+}$ mice

$438 \quad S k i^{\mathrm{G} 34 \mathrm{D} /+}$ mice were generated by homologous recombination. A 10-kb Ski fragment was 439 generated by PCR from mouse embryonic stem cell DNA. The amplicon was subcloned into 
440 pCR2.1-TOPO (Invitrogen Corp.). Site-directed mutagenesis was performed with the In-Fusion

441 HD kit (Clontech Inc.), creating G34D mutation. The Neo cassette was amplified from pMC1neo-

442 polyA vector (Stratagene Inc.) and the fragment containing the Sal1 restriction site and Neo

443 cassette with flanking loxP sequences was subcloned into a unique Sal1 site in the Ski intron after

444 exon 1. All targeting vector sequences, including the sequences of the loxP sites and site-directed

445 mutagenesis-created mutations were confirmed by sanger sequencing. The vector was linearized

446 using a unique (EcoR1) site and electroporated into R1 embryonic stem cells. Positive clones were

447 identified by PCR test. Positives clones were injected into 129S6/ScEvTac blastocysts at

448 embryonic day 3.5 and transferred into pseudopregnant females. Chimeric offspring were mated

449 to $\mathrm{C} 57 \mathrm{BL} / 6 \mathrm{~J}$ mice, and germline transmission was observed for at least 3 independent targeting

450 events for mutant genotype. All exons encompassed by and immediately flanking the targeting

451 vector were analyzed by sequencing of PCR-amplified genomic DNA derived from mutant mice,

452 to demonstrate the fidelity of targeting. Mice were genotyped on the basis of creation of a new

453 BamH1 site in correctly targeted mice. Primers used for amplification were 5'-GAGCCCGATCG

454 CACCATGGAA-3' (sense) and 5'-AAGAGATGGTCTCCCCTTCC-3' (antisense). For testing

455 the random insertion of linearized targeted vector, quantitative PCR of the Neo cassette sequence

456 was performed with a previously verified DNA sample, which contained only a single Neo cassette

457 sequence. The lox $P$-flanked Neo cassette was removed by crossing with a Cre deleter strain, either

458 CMV-Cre (B6.C-Tg(CMV-cre)1Cgn/J, \#006054) purchased from the Jackson Laboratory,

459 followed by mating to $\mathrm{C} 57 \mathrm{BL} / 6 \mathrm{~J}$ strain for at least 5 generations for a $\mathrm{C} 57 \mathrm{BL} / 6 \mathrm{~J}$ genetic

460 background, or Prm-Cre (129S/Sv-Tg(Prm-Cre)58Og/J, \#003328) purchased from Jackson

461 Laboratory, followed by mating to a 129S6/ScEvTac strain for at least 5 generations for a

462 129S6/ScEvTac genetic background. The 129S6/ScEvTac genetic background Ski ${ }^{\mathrm{G} 34 \mathrm{D} /+}$ mice 
463 were used for analyses of dermal, skeletal, and cardiovascular phenotypes. The C57BL/6J genetic

464 background $S k i^{\text {G34D/+ }}$ mice were used for behavioral phenotype analysis. Ski ${ }^{\text {G34D/+:Neo }}$ mice were

465 bred to the 129S6/ScEvTac strain for at least 5 generations, without deletion of the Neo cassette

466 as a separate mouse line. Complete concordance of phenotype for 3 or 2 independent lines and

467 backcrossing to each congenic inbred strain for at least 5 generations excluded any major off-target

468 effects.

469

$470 \quad$ Mouse drug treatment

471 Losartan was dissolved in drinking water and filtered to reach a concentration of $0.5 \mathrm{~g} / \mathrm{L}$,

472 giving an estimated daily dose of $50 \mathrm{mg} / \mathrm{kg} /$ day (based on a 30 -g mouse drinking $3 \mathrm{mLs}$ per day).

473 Atenolol was dissolved in drinking water and filtered to reach a concentration of $0.6 \mathrm{~g} / \mathrm{L}$, giving

474 an estimated daily dose of $60 \mathrm{mg} / \mathrm{kg} /$ day. Placebo-treated mice received drinking water. Mice

475 given these medications were started on treatment at 8 weeks of age and continued for 16 weeks.

476 C646 (Selleckchem, \#S7152) was reconstituted in 10\% DMSO (Sigma) dissolved in PBS, and

477 administered daily by intraperitoneal injection at a dose of $1 \mathrm{mg} / \mathrm{kg} /$ day. Treatment was initiated

478 at 8 weeks of age and continued for 12 weeks. Ten percent DMSO in PBS was administered as a

479 control.

480

481 Mouse echocardiography

482 Nair hair removal cream was used to remove fur from the anterior thorax of the mice the 483 day prior to echocardiography. According to a previous protocol with slight modifications $(7,11$, 484 61), echocardiography was performed on awake, nonsedated mice using a Vevo 2100 imaging 485 system and $40 \mathrm{MHz}$ transducer (Visualsonics). Mice were imaged at 8 weeks of age as a baseline 
486 and every 4 weeks thereafter, until 20 weeks of age. The aorta was imaged in the parasternal long-

487 axis view. Three separate measurements of the maximal internal dimension at the sinus of Valsalva

488 during systole were made in separate cardiac cycles and averaged. All imaging and analysis was

489 performed blinded to genotype and treatment arm.

490

491 Mouse blood pressure

492 According to a previously described protocol with slight modifications $(7,11,61)$, blood

493 pressure was measured by tail-cuff plethysmography using a Harvard Apparatus IITC noninvasive

494 tail cuff device. Mice were placed in a standard acrylic restrainer for adult mice, with an internal

495 diameter of $25 \mathrm{~mm}$ and an adjustable head gate. The end plate was removable, allowing the mice

496 to walk into the restrainer without using force. Hemodynamic recordings were made without

497 sedation or anesthesia. Blood pressure was measured at the end of the drug trial. Mice were

498 habituated for 4 days. On day 5, ten blood pressures were obtained and averaged.

500 Mouse radiography

501 According to a previously described protocol with slight modifications $(7,11,61)$, Mice

502 were anesthetized using a combination of $50 \mathrm{mg} / \mathrm{kg}$ of ketamine- $\mathrm{HCl}$ and $5 \mathrm{mg} / \mathrm{kg}$ xylazine- $\mathrm{HCl}$

503 by intraperitoneal injection before X-ray imaging. Mice were placed in the left lateral decubitus

504 position on a radiolucent platform with a metal paper clip as a scale bar and imaged at $1 x$

505 magnification using a Faxitron MX20 (Faxitron).

506

507 Mouse aorta histological analysis 

infused heart and aorta were removed from body and transected just below the level of the aortic

510 annulus, and just above the aortic root, and 2- to 3-mm transverse sections were mounted in $4 \%$

511 agar prior to fixation in paraffin. Five-micron aortic sections underwent Verhoeff-van Giesen

512 (VVG) and Masson's trichrome staining and were imaged at 40x magnification using a Nikon

513 Eclipse E400 microscope. Aortic wall thickness was measured of 4 sites of 4 representative

514 sections for each mouse. The disruptions of elastin fiber architecture were counted in 4 sections

515 every 25 microns from the aortic annulus. All analyses were performed blinded to genotype and 516 treatment arm and the results were averaged.

Mouse behavioral tests

Behavioral tests were performed per the manufacturer's instructions. All of behavioral tests

520 were performed at 10 weeks of age. For the open field test, a mouse was placed into a corner of a

$52145 \times 45$ cm open-filed chamber (San Diego Instruments) with a 16 x 16 photobeam configuration.

522 The behavior of the mouse was monitored for 5 minutes in each of 6 cycles (total 30 minutes).

523 Mouse activity in center area and/or peripheral areas was recorded by beam interruptions. Total

524 activity was calculated by adding up all beam interruptions during the cycle. Activity in the central

525 and peripheral areas was calculated by adding up the beam interruptions in these two areas,

526 respectively. For the rotarod test, mice were placed on a horizontally oriented, rotating cylinder

527 (rod) suspended above the cage floor (Rotamex-5 rotarod, Columbus Instruments). The

528 acceleration started at $0 \mathrm{rpm}$ and was increased by $1.0 \mathrm{rpm}$ every 5 seconds. Velocity and time

529 were recorded at the time of falling for 3 measurements. Each mouse was given 3 minutes of rest 
530 time between trials. All analyses were performed blinded to genotype and the results were 531 averaged.

533 Human cell culture

534 Primary human dermal fibroblasts were derived from forearm skin biopsies of 2 controls

535 and 2 patients with SGS, as previously described in (21). The fibroblasts were cultured in

536 Dulbecco's modified eagle medium (DMEM) with 10\% fetal bovine serum (FBS) in the presence

537 of antibiotics and passaged confluence. According to a previously described protocol with slight

538 modifications (21), all cell culture experiments were conducted in serum-starved media for 24

539 hours prior to drug treatment. Stimulation was performed using $10 \mathrm{ng} / \mathrm{ml}$ recombinant human

540 TGF $\beta 1$ (R\&D system). C646 dissolved in DMSO was treated at a dose of $20 \mu \mathrm{M}$ for 24 hours of

541 pretreatment before TGF $\beta 1$ stimulation. SD208 dissolved in DMSO was treated at a dose of 10

$542 \mu \mathrm{M}$ for 24 hours of pretreatment before TGF $\beta 1$ stimulation. Cells were collected at baseline, 6

543 hours after TGF $\beta 1$ stimulation. The RNA was extracted from the cells using TRizol

544 (ThermoFisher) via an RNeasy mini kit (QIAGEN), per the manufacturer's instructions.

546 Quantitative RT-PCR expression analysis

547 Total RNA was isolated from mouse aortas or cultured cells using an RNeasy mini kit

548 (QIAGEN), per the manufacturer's instructions. Quantitative PCR was performed in triplicate with

549 TaqMan Universal PCR Master Mix using an ABI Prism 7900 HT QPCR machine (all from

550 Applied Biosystems), per the manufacturer's instructions. The following prevalidated TaqMan

551 probes were used to detect specific transcripts: Mm00801666_g1 (Colla1), Mm01254476_m1

552 (Col3A1), Mm01256744_m1 (Fn1), Mm04205640_g1 (Cdkn1a), Mm01192932_g1 (Ctgf), and 
553 Mm00435860_m1 (Serpine1), Mm00448744_m1（Ski), Mm00456917_m1 (Skil),

554 Mm00484742_m1 (Smad7) and Mm00607939_s1 (Actb) (Life Technologies). For human samples,

555 the following probes were used: Hs00943809_m1 (COL3A1), Hs00365052_m1 (FN1),

556 Hs00161707_m1 (SKI), Hs01045418_m1 (SKIL) and Hs01060665_g1 (ACTB). Reactions were

557 run in triplicate, and relative quantification for each transcript was obtained by normalization

558 against a housekeeping control transcript, such as $\beta-A C T I N(A C T B)$, according to the formula 2-

$559 \mathrm{Ct} / 2^{-\mathrm{Ct}(\mathrm{ACTB})}$.

560

561 Immunofluorescence

562 Immunofluorescence was performed as previous described $(8,9)$. Frozen 10- $\mu \mathrm{M}$ long-axis-

563 view sections were obtained with a cryostat and mounted on glass slides. Sections were dried at

564 room temperature overnight prior to staining. Sections were permeabilized in staining buffer (PBS

565 containing $0.1 \%$ Triton-X 100) for 30 minutes and then incubated with Fc Receptor Block from

566 Innovex for 30 minutes at room temperature, washed briefly in staining buffer, and then incubated

567 again in blocking solution (0.1\% Triton-X 100, 1:50 goat serum, 0.3M glycine) for 30 minutes.

568 Primary antibodies were diluted at 1:100 in staining buffer and incubated with goat anti-rabbit

569 secondary antibody conjugated to Alexa Fluor 555 (Life Technologies) at 1:200 for 1 hour before

570 being mounted with VECTASHIELD Hard Set Mounting Medium with DAPI. Images were

571 acquired on a Zeiss Axio Examiner with a 710NLO-Meta multiphoton confocal microscope at 25x

572 magnification. The following primary antibody was used: anti-H3K27ac (Abcam, ab4729).

573

574 Statistics 
All quantitative data are shown as bar graphs, produced using Graphpad Prism. Mean \pm

576 standard errors of the mean (SEM) are displayed. Statistical analyses were performed using non-

577 parametric test (Kruskal-Wallis test with Dunn's multiple comparison test). A $P$ value $<0.05$ was

578 considered to be statistically significant for all tests.

579

580 Study approval

581 This study was performed in accordance with the recommendations in the Guide for the

582 Care and Use of Laboratory Animals of the National Institutes of Health. All of the animals were

583 handled according to approved institutional animal care and use committee (IACUC) protocols

584 of the Johns Hopkins University School of Medicine. The protocol was approved by the

585 Committee on the Ethics of Animal Experiments of the Johns Hopkins University School of

586 Medicine.

587 


\section{Author contributions}

H.C.D and B.E.K. developed the concept. B.E.K. generated mouse models, designed,

590 performed and directed experiments, analyzed data and wrote the manuscript. J.J.D., E.G.M., and

591 H.C.D. aided in experimental design and interpretation of the data. R.B. aided in mouse colony

592 maintenance and drug treatment. D.B. aided in mouse echocardiography and analyses. R.B., J.J.D.,

593 E.G.M., and H.C.D provided essential expertise in the editing of the manuscript. All authors

594 discussed the results and commented on the manuscript prior to submission.

595

596 Acknowledgements

597 This work was supported by the Howard Hughes Medical Institute, the Johns Hopkins

598 University School of Medicine Cellular and Molecular Medicine graduate training program

599 (T32GM008752). We thank the Johns Hopkins University School of Medicine Transgenic Mouse

600 Core laboratory for expert technical assistance with generating mouse models; J. Habashi for

601 echocardiography analysis; the Johns Hopkins Hospital Department of Pathology Reference

602 laboratory for histology; the Johns Hopkins University School of Medicine Behavioral Core

603 laboratory for mouse behavioral testing.

604 


\section{References}

606

607

608

609

610

611

612

613

614

615

616

617

618

619

620

621

622

623

624

625

626

627

628

629

630

631

632

633

634

635

1. Schepers D et al. The SMAD-binding domain of SKI: a hotspot for de novo mutations causing Shprintzen\&ndash;Goldberg syndrome. Eur J Hum Genet 2014;23(2):1-5.

2. Pagon RA et al. Shprintzen-Goldberg Syndrome1993;

3. Dietz HC et al. Marfan syndrome caused by a recurrent de novo missense mutation in the fibrillin gene. Nature 1991;352(6333):337-339.

4. Loeys BL et al. A syndrome of altered cardiovascular, craniofacial, neurocognitive and skeletal development caused by mutations in TGFBR1 or TGFBR2. Nat Genet 2005;37(3):275281.

5. Neptune ER et al. Dysregulation of TGF-beta activation contributes to pathogenesis in Marfan syndrome. Nat Genet 2003;33(3):407-411.

6. Judge DP et al. Evidence for a critical contribution of haploinsufficiency in the complex pathogenesis of Marfan syndrome. J. Clin. Invest. 2004;114(2):172-181.

7. Holm TM et al. Noncanonical TGF Signaling Contributes to Aortic Aneurysm Progression in Marfan Syndrome Mice. Science 2011;332(6027):358-361.

8. Gallo EM et al. Angiotensin II-dependent TGF- $\beta$ signaling contributes to Loeys-Dietz syndrome vascular pathogenesis. J. Clin. Invest. 2014;124(1):448-460.

9. Habashi JP et al. Losartan, an AT1 Antagonist, Prevents Aortic Aneurysm in a Mouse Model of Marfan Syndrome. Science 2006;312(5770):117-121.

10. Cohn RD et al. Angiotensin II type 1 receptor blockade attenuates TGF- $\beta$-induced failure of muscle regeneration in multiple myopathic states. Nat Med 2007;13(2):204-210.

11. Habashi JP et al. Angiotensin II Type 2 Receptor Signaling Attenuates Aortic Aneurysm in Mice Through ERK Antagonism. Science 2011;332(6027):361-365.

12. MacFarlane EG et al. Lineage-specific events underlie aortic root aneurysm pathogenesis in Loeys-Dietz syndrome. J. Clin. Invest. 2019;129(2):659-675.

13. Chen $\mathrm{X}$ et al. Conundrum of angiotensin II and TGF- $\beta$ interactions in aortic aneurysms. Curr Opin Pharmacol 2013;13(2):180-185.

14. Chen $X$ et al. TGF- $\beta$ Neutralization Enhances AngII-Induced Aortic Rupture and Aneurysm in Both Thoracic and Abdominal Regions. PLoS ONE 2016;11(4):e0153811.

15. Wang $Y$ et al. TGF-beta activity protects against inflammatory aortic aneurysm progression and complications in angiotensin II-infused mice. J. Clin. Invest. 2010;120(2):422-432. 
16. Loeys BL et al. Aneurysm syndromes caused by mutations in the TGF-beta receptor. $N$. Engl. J. Med. 2006;355(8):788-798.

638 17. Lindsay ME et al. Loss-of-function mutations in TGFB2 cause a syndromic presentation of thoracic aortic aneurysm. Nature Publishing Group 2012;44(8):922-927.

18. Regalado ES et al. Exome sequencing identifies SMAD3 mutations as a cause of familial thoracic aortic aneurysm and dissection with intracranial and other arterial aneurysms. Circ. Res.

642 2011;109(6):680-686.

643 19. Tan CK et al. SMAD3 deficiency promotes inflammatory aortic aneurysms in angiotensin II644 infused mice via activation of iNOS. J Am Heart Assoc 2013;2(3):e000269.

645

20. Dai X et al. SMAD3 deficiency promotes vessel wall remodeling, collagen fiber reorganization and leukocyte infiltration in an inflammatory abdominal aortic aneurysm mouse model. Sci Rep 2015;5(1):10180.

21. Doyle AJ et al. Mutations in the TGF- $\beta$ repressor SKI cause Shprintzen-Goldberg syndrome with aortic aneurysm. Nature Publishing Group 2012;44(11):1249-1254.

22. Carmignac V et al. In-Frame Mutations in Exon 1 of SKI Cause Dominant ShprintzenGoldberg Syndrome. The American Journal of Human Genetics 2012;91(5):950-957.

23. Akiyoshi S et al. c-Ski acts as a transcriptional co-repressor in transforming growth factorbeta signaling through interaction with smads. J. Biol. Chem. 1999;274(49):35269-35277.

24. Luo K et al. The Ski oncoprotein interacts with the Smad proteins to repress TGFbeta signaling. Genes \& Development 1999;13(17):2196-2206.

25. Stroschein SL et al. Negative feedback regulation of TGF-beta signaling by the SnoN oncoprotein. Science 1999;286(5440):771-774.

26. Sun Y et al. Interaction of the Ski oncoprotein with Smad3 regulates TGF-beta signaling. Mol. Cell 1999;4(4):499-509.

27. Xu W et al. Ski acts as a co-repressor with Smad2 and Smad3 to regulate the response to type beta transforming growth factor. Proc. Natl. Acad. Sci. U.S.A. 2000;97(11):5924-5929.

28. Wu JW et al. Structural mechanism of Smad4 recognition by the nuclear oncoprotein Ski: insights on Ski-mediated repression of TGF-beta signaling. Cell 2002;111(3):357-367.

29. Liu X et al. Ski/Sno and TGF-beta signaling. Cytokine Growth Factor Rev. 2001;12(1):1-8.

30. Chen W et al. Competition between Ski and CREB-binding protein for binding to Smad proteins in transforming growth factor-beta signaling. J. Biol. Chem. 2007;282(15):1136511376. 
31. Gori I et al. Mutations in SKI in Shprintzen-Goldberg syndrome lead to attenuated TGF- $\beta$ responses through SKI stabilization. Elife 2021;10. doi:10.7554/eLife.63545

32. Holtwick R et al. Smooth muscle-selective deletion of guanylyl cyclase-A prevents the acute but not chronic effects of ANP on blood pressure. Proc. Natl. Acad. Sci. U.S.A. 2002;99(10):7142-7147.

33. Creyghton MP et al. Histone H3K27ac separates active from poised enhancers and predicts developmental state. Proc. Natl. Acad. Sci. U.S.A. 2010;107(50):21931-21936.

34. Rada-Iglesias A et al. A unique chromatin signature uncovers early developmental enhancers in humans. Nature 2011;470(7333):279-283.

35. Visel A et al. ChIP-seq accurately predicts tissue-specific activity of enhancers. Nature 2009;457(7231):854-858.

36. Wang Q et al. Spatial and temporal recruitment of androgen receptor and its coactivators involves chromosomal looping and polymerase tracking. Mol. Cell 2005;19(5):631-642.

37. Jin Q et al. Distinct roles of GCN5/PCAF-mediated H3K9ac and CBP/p300-mediated H3K18/27ac in nuclear receptor transactivation. EMBO J. 2011;30(2):249-262.

38. Weinert BT et al. Time-Resolved Analysis Reveals Rapid Dynamics and Broad Scope of the CBP/p300 Acetylome. Cell 2018;174(1):231-244.e12.

39. Raisner R et al. Enhancer Activity Requires CBP/P300 Bromodomain-Dependent Histone H3K27 Acetylation. CellReports 2018;24(7):1722-1729.

40. Inoue $\mathrm{Y}$ et al. Smad3 is acetylated by p300/CBP to regulate its transactivation activity. Oncogene 2007;26(4):500-508.

41. Bowers EM et al. Virtual ligand screening of the p300/CBP histone acetyltransferase: identification of a selective small molecule inhibitor. Chem Biol 2010;17(5):471-482.

42. Kaartinen V, Warburton D. Fibrillin controls TGF-beta activation. Nat Genet 2003;33(3):331-332.

43. Cannaerts E et al. TGF- $\beta$ signalopathies as a paradigm for translational medicine. Eur J Med Genet 2015;58(12):695-703.

44. Ramirez F et al. Fibrillin microfibrils: multipurpose extracellular networks in organismal physiology. Physiol Genomics 2004;19(2):151-154.

45. Bertoli-Avella AM et al. Mutations in a TGF- $\beta$ ligand, TGFB3, cause syndromic aortic aneurysms and dissections. J. Am. Coll. Cardiol. 2015;65(13):1324-1336.

46. Courtois A et al. A novel SMAD3 mutation caused multiple aneurysms in a patient without osteoarthritis symptoms. Eur J Med Genet 2017;60(4):228-231. 
47. Micha D et al. SMAD2 Mutations Are Associated with Arterial Aneurysms and Dissections. Hum. Mutat. 2015;36(12):1145-1149.

48. Robinson PN et al. Shprintzen-Goldberg syndrome: fourteen new patients and a clinical analysis. Am. J. Med. Genet. 2005;135(3):251-262.

49. Zhang B et al. A dynamic H3K27ac signature identifies VEGFA-stimulated endothelial enhancers and requires EP300 activity. Genome Research 2013;23(6):917-927.

50. Yuan $\mathrm{H}$ et al. Involvement of p300/CBP and epigenetic histone acetylation in TGF- $\beta 1$ mediated gene transcription in mesangial cells. Am. J. Physiol. Renal Physiol. 2013;304(5):F601-13.

51. Petruk S et al. Stepwise histone modifications are mediated by multiple enzymes that rapidly associate with nascent DNA during replication. Nat Commun 2013;4:2841.

52. Dias JD et al. Methylation of RNA polymerase II non-consensus Lysine residues marks early transcription in mammalian cells. Elife 2015;4:387.

53. Huang W-S et al. CIL-102-Induced Cell Cycle Arrest and Apoptosis in Colorectal Cancer Cells via Upregulation of p21 and GADD45. PLoS ONE 2017;12(1):e0168989.

54. Chen $\mathrm{G}$ et al. SREBP-1 is a novel mediator of TGF $\beta 1$ signaling in mesangial cells. $J$ Mol Cell Biol 2014;6(6):516-530.

55. Santer FR et al. Inhibition of the acetyltransferases p300 and CBP reveals a targetable function for p300 in the survival and invasion pathways of prostate cancer cell lines. Mol. Cancer Ther. 2011;10(9):1644-1655.

56. Marek R et al. Paradoxical enhancement of fear extinction memory and synaptic plasticity by inhibition of the histone acetyltransferase p300. J. Neurosci. 2011;31(20):7486-7491.

57. Maddox SA et al. p300/CBP histone acetyltransferase activity is required for newly acquired and reactivated fear memories in the lateral amygdala. Learn. Mem. 2013;20(2):109-119.

58. $\mathrm{Su} \mathrm{H}$ et al. Histone Acetyltransferase p300 Inhibitor Improves Coronary Flow Reserve in SIRT3 (Sirtuin 3) Knockout Mice. J Am Heart Assoc 2020;9(18):e017176.

59. Reddy MA et al. Losartan reverses permissive epigenetic changes in renal glomeruli of diabetic db/db mice. Kidney Int. 2014;85(2):362-373.

60. Hayashi K et al. Renin-angiotensin blockade resets podocyte epigenome through Kruppellike Factor 4 and attenuates proteinuria. Kidney Int. 2015;88(4):745-753.

61. Doyle JJ et al. A deleterious gene-by-environment interaction imposed by calcium channel blockers in Marfan syndrome. Elife 2015;4:e81743. 
A

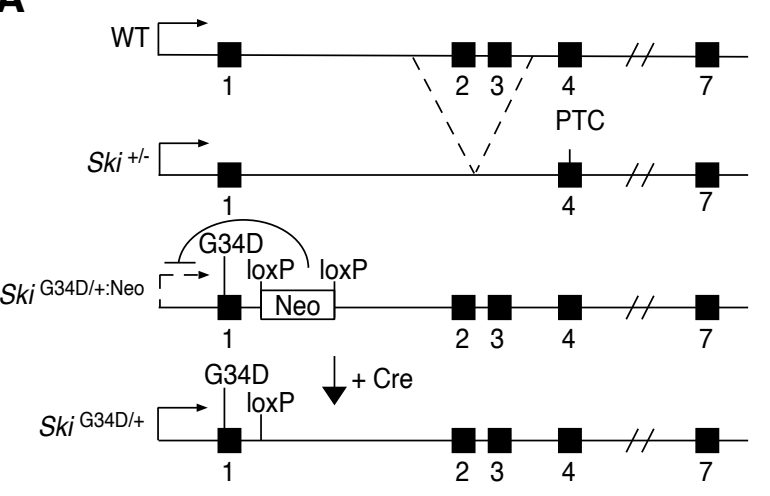

C

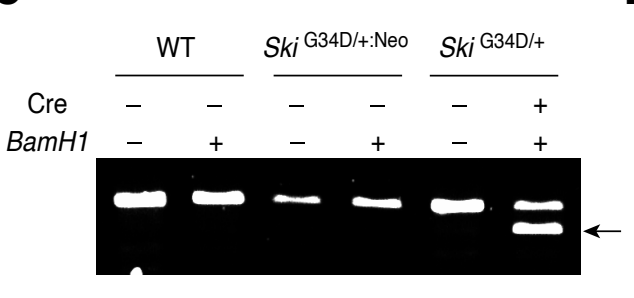

E
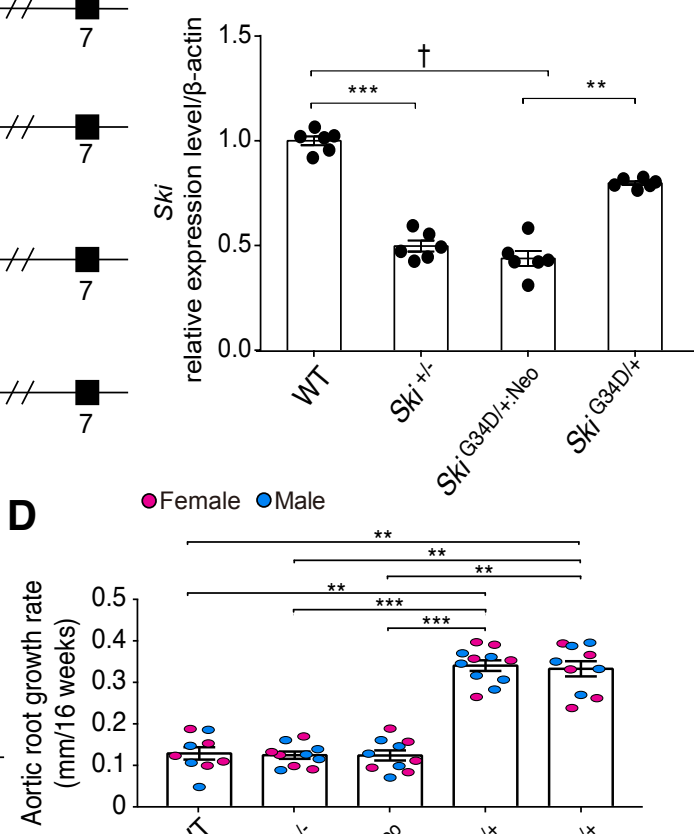

B

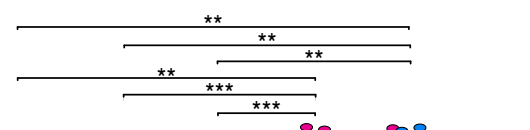

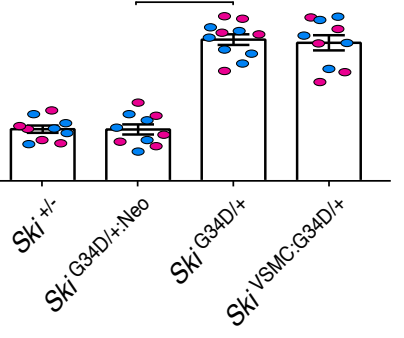

$\mathbf{F}$
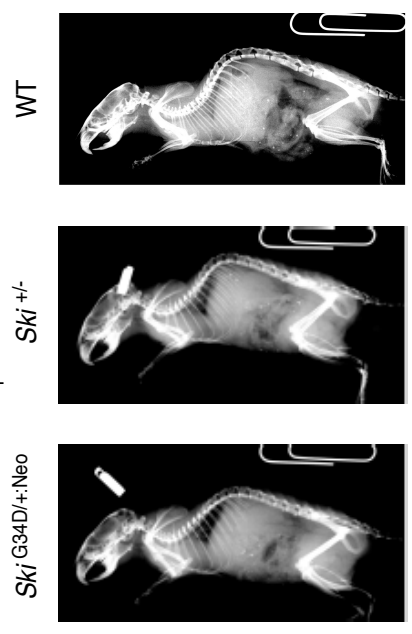

+
के
क
क

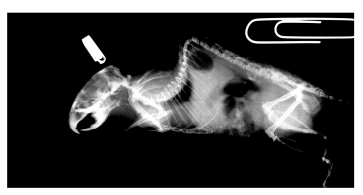

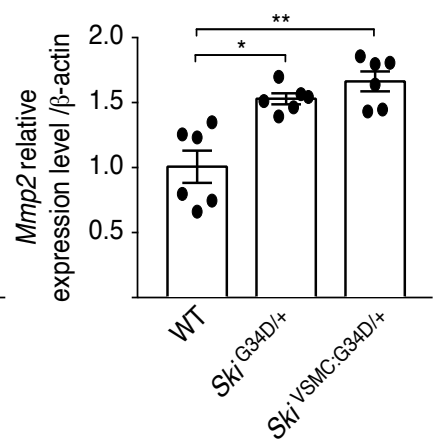
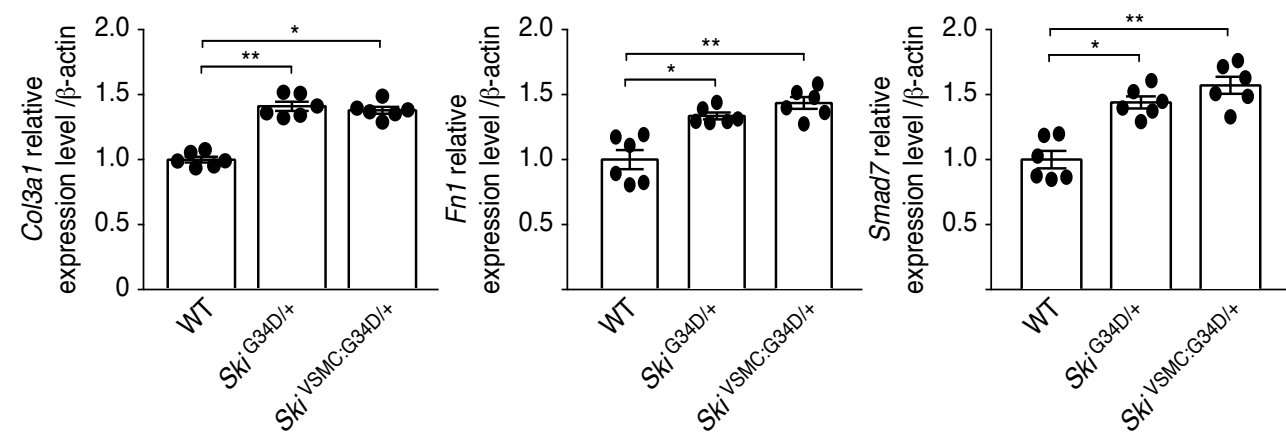

Figure 1. Characterization of SGS mouse models. (A) Structure of Ski haploinsufficient and Ski mutant alleles in mouse models. Deletion of exons 2 and 3 of Ski creates a premature termination codon (PTC) in exon 4 of the Ski haploinsufficient allele. (B) Mean expression level ( \pm SEM) of Ski mRNA in the tail of each mouse line by qPCR. Both $S_{k i}{ }^{+/-}$and $S k i G 34 \mathrm{D} /+$ :Neo haploinsufficient lines express half the normal complement of Ski mRNA, while the $S k i^{\mathrm{G} 34 \mathrm{D} /+}$ mouse line expresses levels comparable to controls; $\mathrm{n}=6$ per group. (C) Expression of WT and mutant $S k i$ in each mouse line. The $S k i^{G 34 D /+}$ mouse line uniquely expresses the mutant allele, as shown by the BamH1 restriction fragment of amplified cDNA. (D) Mean

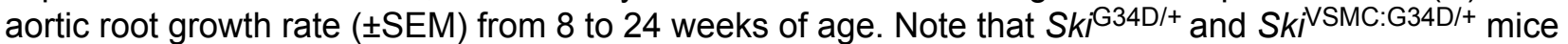
had significantly greater aortic growth compared to other groups; wild type $(n=9), S k i^{+/-}(n=10)$, $S k i^{G 34 D /+:-}$ $\mathrm{Neo}(n=10)$, SkiG34D/+ $(n=11)$, SkiVSMC:G34D/+ $(n=10)$. (F) Skeletal phenotype: representative spine radiographs for each mouse line at 24 weeks of age. Compared to other mouse lines, only SkiG34D/+ mice demonstrated skeletal deformities. (G) Expression levels of TGF $\beta$ target genes (Co/3a1, Fn1, Smad7 and $M m p 2)$ relative to $\beta$-actin control and normalized to WT expression ( $\pm S E M)$, as determined by qPCR. Compared with WT littermates, SkiG34D/+ mice and SkiVSMC:G34D/+ mice demonstrated increased target gene expression; $n=6$ for all groups. Non-parametric Kruskal-Wallis test with Dunn's multiple comparison test was used to assess for statistical significance between comparing groups. For all graphs, each bar defines the median with standard error indicated by whiskers and numerical data are presented as scatter dot-plots. ${ }^{*} \mathrm{P}<0.05 ;{ }^{* *} \mathrm{P}<0.01 ;{ }^{* * *} \mathrm{P}<0.001 ; \dagger \mathrm{P}<10^{-4}$. 
A

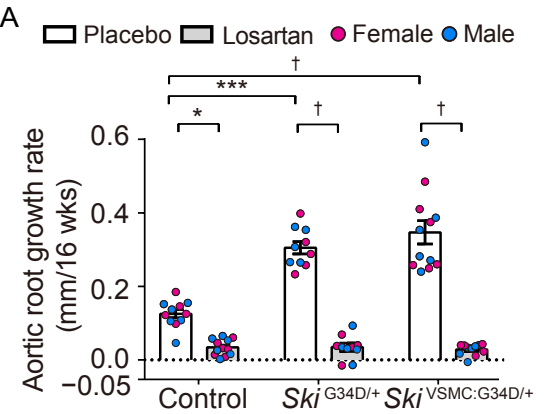

C

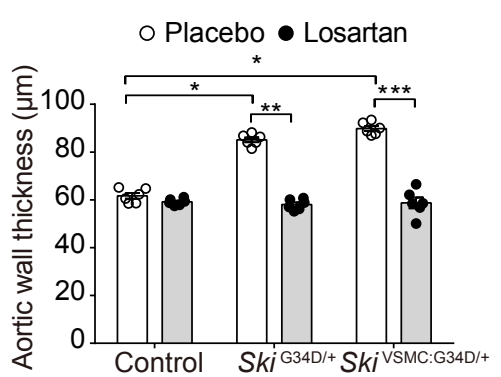

D

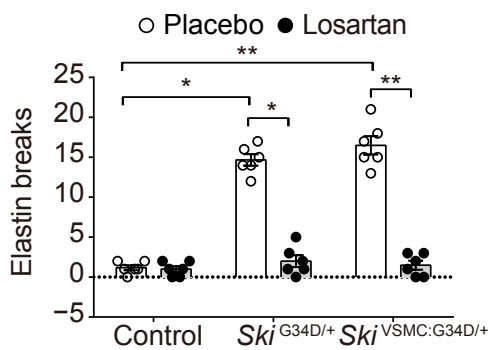

B

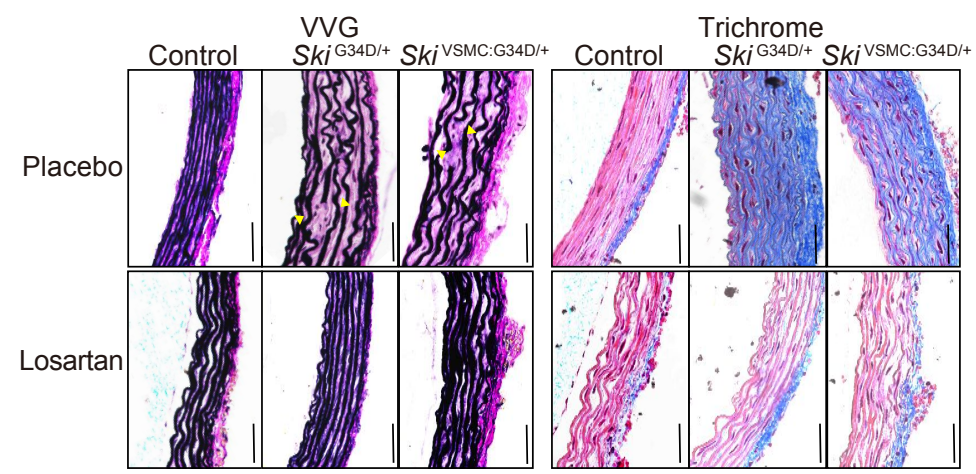

$\mathrm{E}$
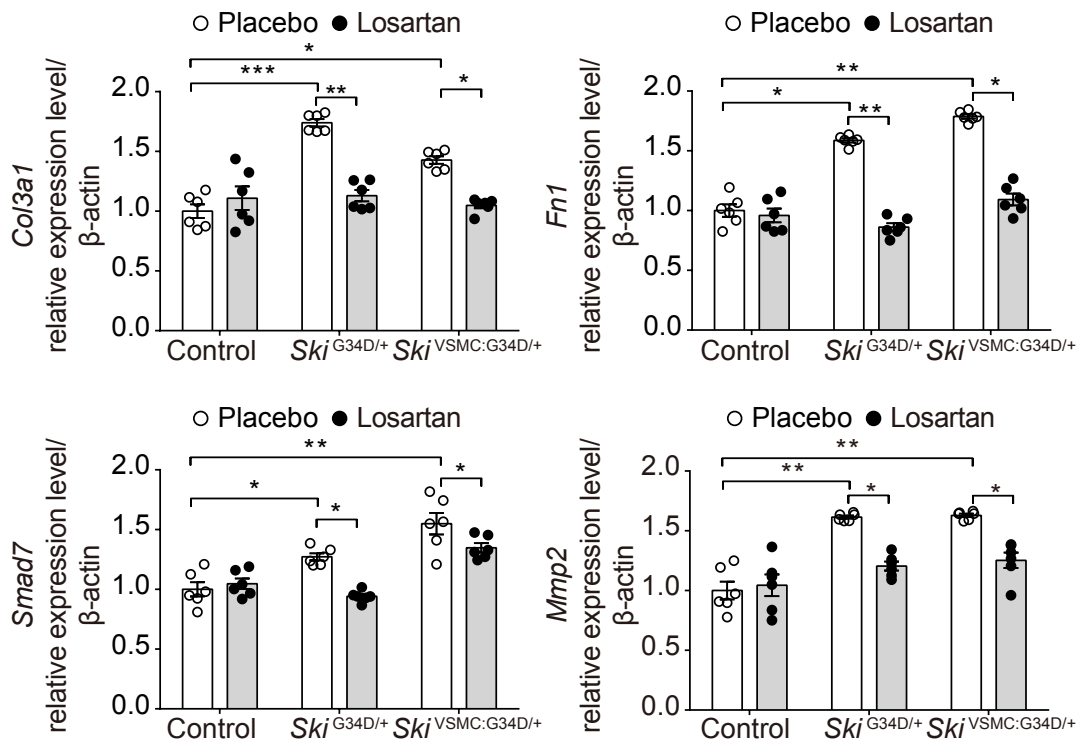

Figure 2. Therapeutic effect of losartan on aortic aneurysm in SGS mice. (A) Mean aortic root growth $( \pm S E M)$ over 16 weeks of treatment with losartan. Compared with placebo-treated $S k i^{\mathrm{G} 34 \mathrm{D} /+}$ and Ski ${ }^{\text {VSMC:G34D/+ }}$ mice, losartan-treated SkiG34D/+ and Ski ${ }^{\mathrm{VSMC}: G 34 \mathrm{D} /+}$ mice demonstrated reduced aortic root growth. Control-placebo $(n=11)$, SkiG34D/+_placebo $(n=10)$, SkivSMC:G34D/+_placebo $(n=12)$; Control-losartan $(n=12)$, Ski ${ }^{\mathrm{G} 34 \mathrm{D} /+}$-losartan $(\mathrm{n}=10)$, Ski $\mathrm{V}^{\mathrm{VSMC}: \mathrm{G} 34 \mathrm{D} /+}$ mice-losartan $(\mathrm{n}=11)$. (B) Representative cross-sections of the aortic root, stained with VVG (left) and Masson's trichrome (right), in placebo- and losartan-treated SGS mice; yellow arrow indicates elastic fiber fragmentation. Black line indicates scale bar $(50 \mu \mathrm{m})$. (C) Aortic wall thickness ( \pm SEM). Compared with controls, placebo-treated SkiG34D/+ and Ski ${ }^{\mathrm{VSMC}: G 34 \mathrm{D} /+}$ mice demonstrated greater aortic wall thickening, which was significantly reduced in losartan-treated animals; $n=6$ in all groups. (D) Extent of aortic wall elastic fiber damage, measured by number of elastin breaks $( \pm S E M)$. Compared with controls, placebo-treated $S k i^{\mathrm{G} 34 \mathrm{D} /+}$ and $S k^{\mathrm{vSMC}: \mathrm{G} 3 \mathrm{D} /+}$ mice demonstrated increased elastin breaks, which was significantly reduced in losartan-treated animals; $n=6$ per group. (E) TGF $\beta$ target gene expression relative to $\beta$-actin and normalized to control expression ( $\pm S E M)$. Compared with controls, SkiG34D/+ and SkivSMc:G34D/+ mice demonstrated increased TGF $\beta$ target gene expression, which was significantly reduced in losartan-treated animals; $n=6$ per group. Non-parametric Kruskal-Wallis test with Dunn's multiple comparison test was used to assess for statistical significance between comparing groups. For all graphs, each bar defines the median with standard error indicated by whiskers and numerical data are presented as scatter dot-plots. ${ }^{*} \mathrm{P}<0.05 ;{ }^{* \star} \mathrm{P}<0.01 ;{ }^{* \star *} \mathrm{P}<0.001 ; \dagger \mathrm{P}<10^{-4}$. 
A

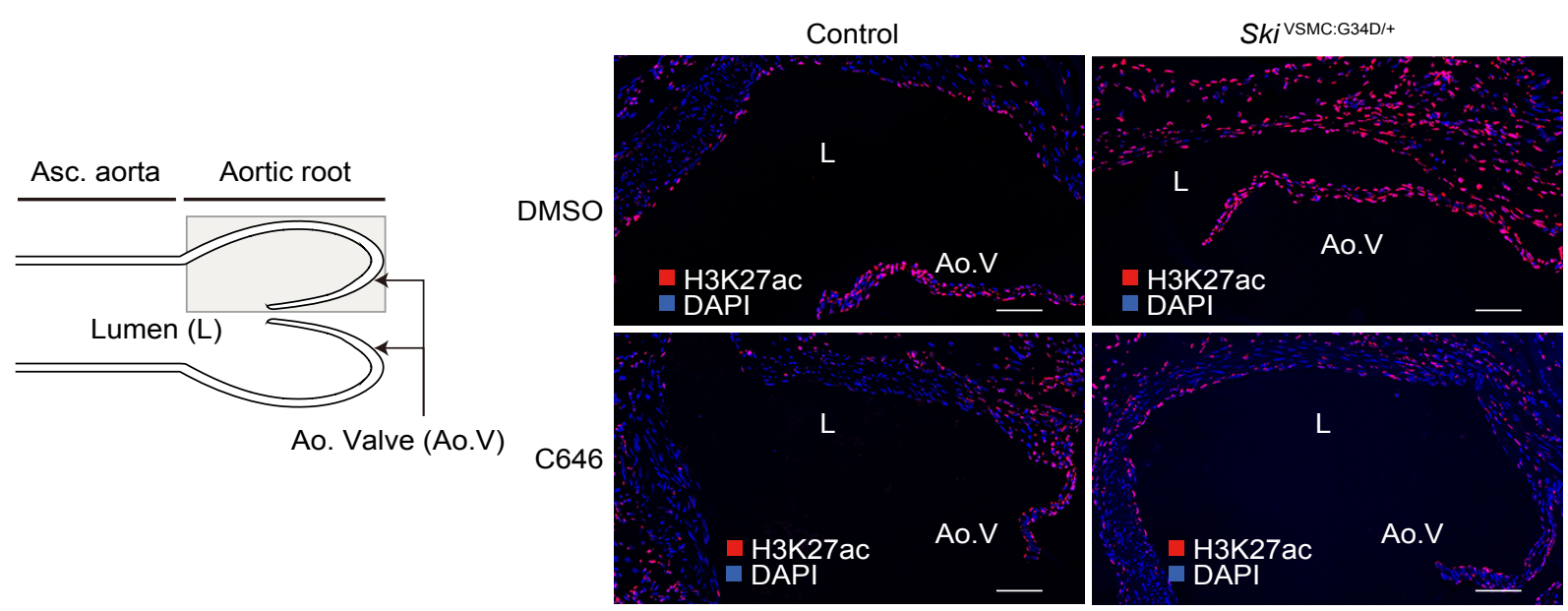

B

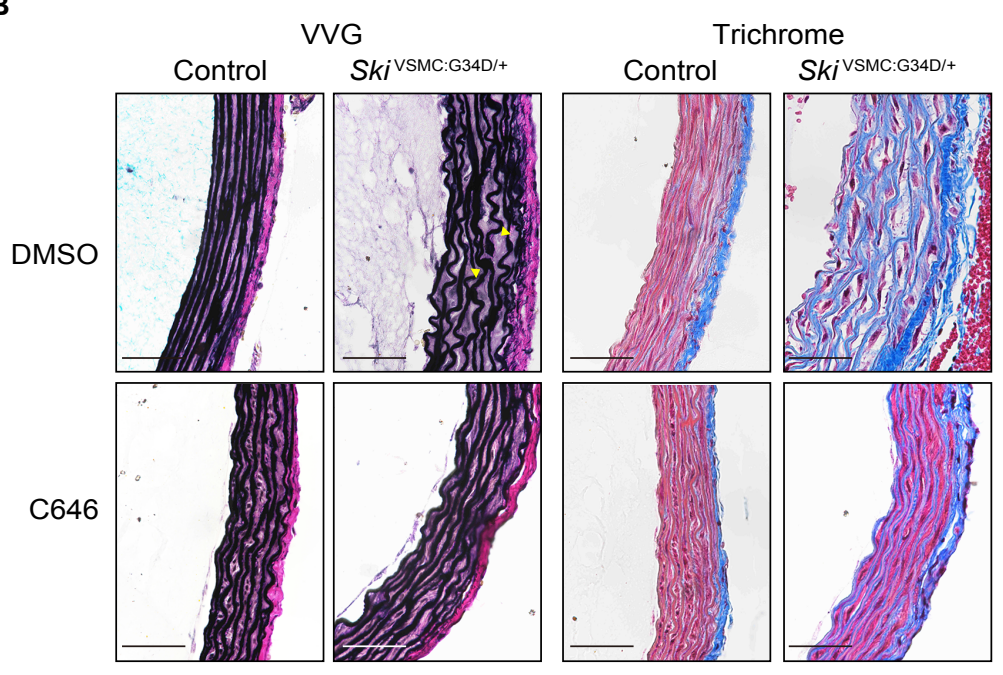

C

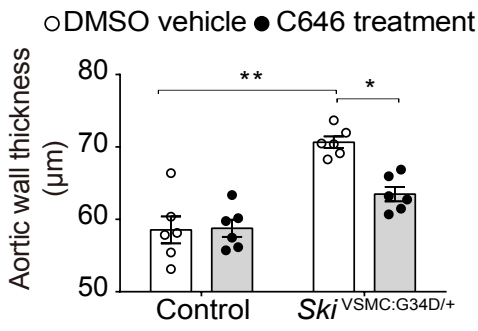

D

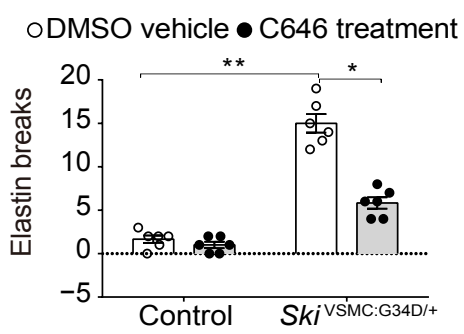

Figure 3. Phenotypic effect of CBP/P300 inhibition using C646 on aortic root aneurysm in mouse models of SGS. (A) Representative immunofluorescence image of aortic root samples of control and Ski ${ }^{\mathrm{VSMC}: \mathrm{G} 3 \mathrm{D} /+}$ mice treated with either $\mathrm{C} 646$ or vehicle (DMSO) probed for histone 3 lysine 27 acetylation (H3K27ac). Compared with controls, SkivMc:G34D/+ mice demonstrated increased acetyla-

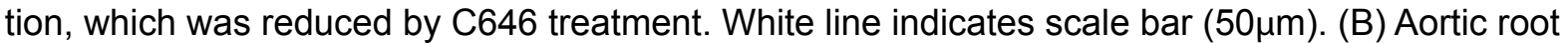
histology in DMSO- and C646-treated control and SkV VSMC:G34D/+ mice. Images show representative cross-sections stained with VVG (left) and Masson's trichrome stain (right). Yellow arrows indicate elastic fiber breaks. Black line indicates scale bar $(50 \mu \mathrm{m})$. (C) Aortic wall thickness ( \pm SEM) in DMSOand C646-treated control and SGS mice. Compared with controls, DMSO-treated SkivSMC:G34D/+ animals showed greater medial wall thickening, which was reduced in C646-treated SkiSMc:G34D/+ littermates; $n=6$ for all groups. (D) Mean number of elastin breaks ( \pm SEM) in DMSO- and C646-treated control and SGS mice. Compared with controls, DMSO-treated Ski ${ }^{\mathrm{VSMC}: G 34 \mathrm{D} /+}$ mice showed more elastin breaks, which was prevented by $\mathrm{C} 646$ treatment; $n=6$ for all groups. Non-parametric Kruskal-Wallis test with Dunn's multiple comparison test was used to assess for statistical significance between comparing groups. For all graphs, each bar defines the median with standard error indicated by whiskers and numerical data are presented as scatter dot-plots. ${ }^{*} \mathrm{P}<0.05 ;{ }^{* *} \mathrm{P}<0.01$. 
A

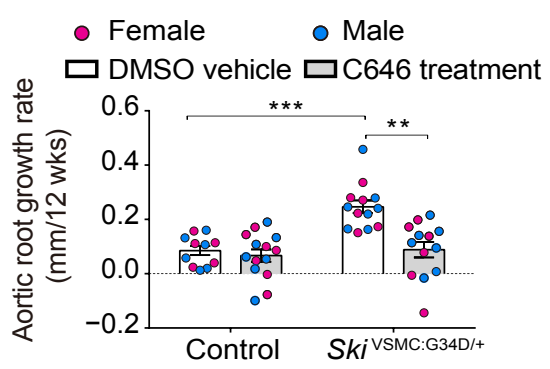

C

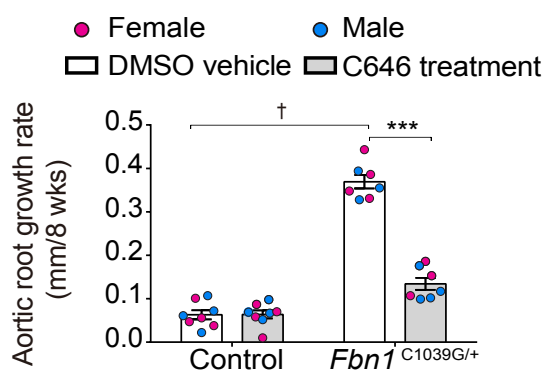

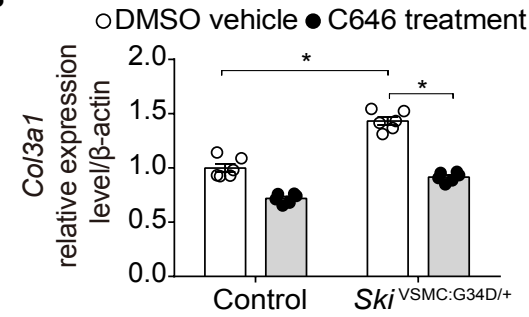

oDMSO vehicle $\bullet$ C646 treatment

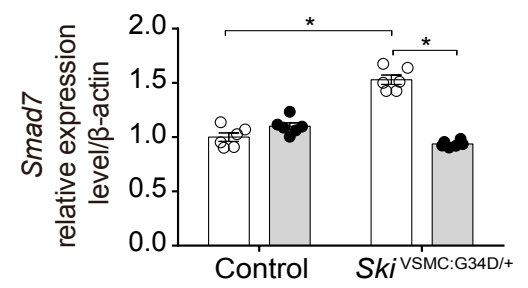

D

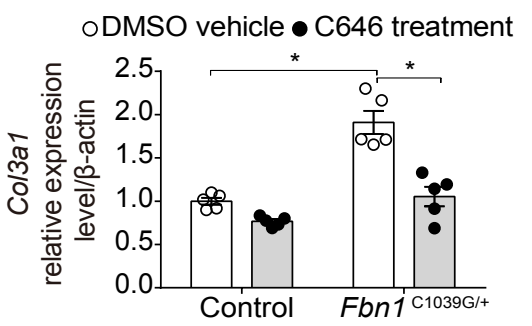

oDMSO vehicle $\bullet$ C646 treatment

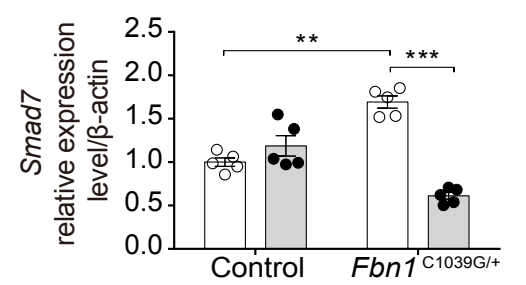

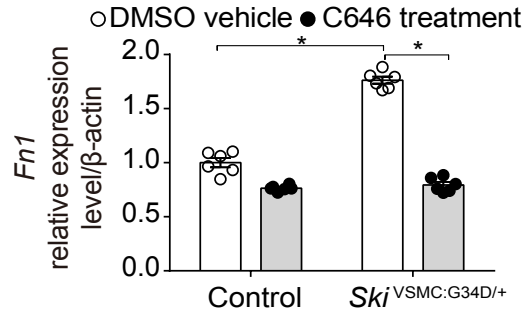

oDMSO vehicle $\bullet$ C646 treatment

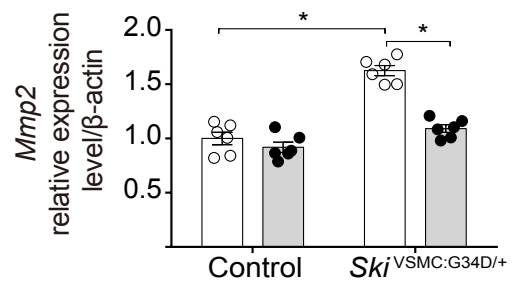

oDMSO vehicle $\bullet$ C646 treatment

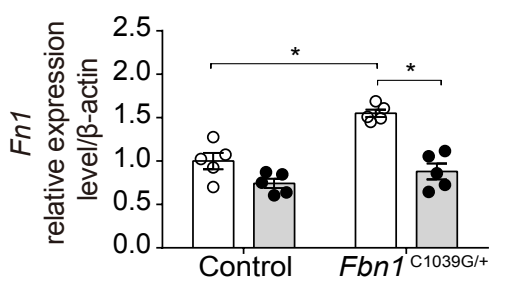

oDMSO vehicle $\bullet$ C646 treatment

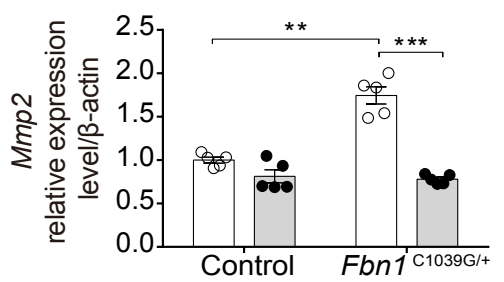

Figure 4. Therapeutic effect of CBP/P300 inhibition using C646 on aortic root aneurysm in mouse models of SGS and MFS. (A) Mean aortic root growth over 12 weeks of treatment with C646 in control and $S k{ }^{v S M C: G 34 D /+}$ mice. Compared with controls, $S k{ }^{v S M C: G 34 D /+}$ mice demonstrated significantly greater aortic root growth, which was reduced by treatment with C646; Control-DMSO $(n=11)$, SkivSMC:G34D/+_DMSO ( $n=13)$; Control-C646 ( $n=14)$, SkivSMC:G34D/+_C646 $(n=13)$. (B) TGF $\beta$ target gene expression relative to $\beta$-actin and normalized to control expression $( \pm S E M)$, as determined by qPCR. Compared with controls, $S k v^{\mathrm{VSMC}: G 34 \mathrm{D} /+}$ mice showed increased target gene expression, which was reduced by $\mathrm{C} 646$ treatment; $n=6$ per group. (C) Mean aortic root growth over 8 weeks of treatment with C646 in control and MFS $\left(F b n 1^{103934 G /+}\right)$ mice. Compared with DMSO-treated MFS mice, C646-treated MFS animals had a significant reduction in aortic root growth rate; Control-DMSO $(n=8)$, MFS-DMSO $(n=8)$; Control-C646 $(n=7)$, MFS-C646 ( $n=7)$. (D) TGF $\beta$ target gene expression relative to $\beta$-actin and normalized to control expression $( \pm S E M)$, as determined by qPCR. Compared with controls, MFS $\left(F b n 1^{\mathrm{C} 103934 \mathrm{G} /+}\right)$ mice had significantly increased target gene expression, which was reduced in C646-treated MFS animals. Non-parametric Kruskal-Wallis test with Dunn's multiple comparison test was used to assess for statistical significance between comparing groups. For all graphs, each bar defines the median with standard error indicated by whiskers and numerical data are presented as scatter dot-plots. ${ }^{*} \mathrm{P}<0.05 ;{ }^{* *} \mathrm{P}<0.01 ;{ }^{* * *} \mathrm{P}<0.001 ; \mathrm{\dagger} \mathrm{P}<10^{-4}$. 